\title{
Optical Precursors with Electromagnetically Induced Transparency in Cold Atoms
}

\author{
Dong Wei, J.F. Chen, M. M. T. Loy, G. K. L. Wong, and Shengwang Du* \\ Department of Physics, The Hong Kong University of Science and Technology, Clear Water Bay, Kowloon, Hong Kong, China
}

(Received 10 July 2009; published 28 August 2009)

\begin{abstract}
We report the observation of Sommerfeld-Brillouin optical precursors generated from a long squaremodulated laser pulse propagating through a cold atomic ensemble with electromagnetically induced transparency. The optical depth $\left(\alpha_{0} L\right)$ of the medium can be varied from 0 up to 50 . We demonstrated that the step-on rising and step-off falling edges propagate with the speed of light in vacuum without a slow light effect. At high $\alpha_{0} L$, the precursor is separated from the delayed main pulse at the rising edge, while at the falling edge, we observe damped oscillatory structures resulting from the interference between the precursor and main field.
\end{abstract}

PACS numbers: 42.50.Gy, 37.10.Gh

Pulse propagation through a dispersive medium with a complex refractive index $n(\omega)$ is a fundamental problem in classical electromagnetism because it is directly linked to the nonviolation of Einstein's causality [1]. In 1914, Sommerfeld and Brillouin showed theoretically that the front of a step-modulated optical pulse propagating in such media always travels at the light velocity in vacuum $c$ [2]. This front, in the form of a transient wave now known as the Sommerfeld-Brillouin precursor, is then followed by the main pulse traveling at its group velocity. This conclusion, while qualitatively correct, was found to be quantitatively incorrect, particularly on greatly underestimating the amplitude and duration of the precursors [3]. With the intriguing possibility that precursors might be useful as optical probes of biological tissues or in underwater communication [4,5], there has been renewed interest in this problem. Recently a number of theoretical studies have been carried out [6-8], and there are now precise quantitative predictions on the precursor and main pulse, as well as their dependencies on the strength and line shape of the absorber.

Experimentally, precursors have been reported in gamma rays [9], microwave [10], optical [5,11,12], and sound $[13,14]$ waves. While the features are in qualitative agreement with theory, these data were rather limited in both scope and precision, far below what is required to compare with the theoretical predictions. Moreover, the past experimental studies of precursors in classical pulse propagation have always been focused on an opaque medium with single or multiple Lorentz absorption lines. As a result, the precursor signal cannot be separated clearly from the main pulse or otherwise the main field is absorbed. Recently, motivated by the observation of optical precursors at the biphoton level [15], the advantage of using electromagnetically induced transparency (EIT) and slow light $[16,17]$ was pointed out $[6,7]$. In this case, in a single experiment, both the precursor and the main pulse can be observed, and with proper parameters, they can be well separated in time with comparable amplitudes. In this Letter, we report the first observation of optical precursors generated from a long square-modulated laser pulse passing through a three-level EIT system. Our experimental findings are in all cases in excellent agreement with theoretical predictions, over the wide range of optical depth $\left(\alpha_{0} L\right)$ from 0 up to 50 . Since the system parameters are all experimentally determined, this excellent agreement over the wide parameter space is in fact without any adjustable parameters.

The schematics of an EIT three-level $\Lambda$ system and the experimental configuration are shown in Fig. 1. We work with a two-dimensional (2D) ${ }^{85} \mathrm{Rb}$ magneto-optical trap (MOT) with a longitudinal length $L=1.5 \mathrm{~cm}$ and a temperature of about $100 \mu \mathrm{K}$. The atoms are initially prepared in the ground state $|1\rangle$. In the presence of a strong coupling laser beam $\left(\omega_{c}\right)$, a weak square-modulated probe pulse $\left(\omega_{p}\right)$ propagates through the MOT and is detected by a photon-multiplier tube (PMT, Hamamatsu, H6780-20, with $0.78 \mathrm{~ns}$ rise time). The square probe pulse, generated using a $3 \mathrm{GHz}$ acoustic-optical modulator (AOM, Brimrose) controlled by a digital pulse generator (SRS, DG645), has a length of $2 \mu \mathrm{s}$ and a rise (fall) time of $7 \mathrm{~ns}$. The transmitted waveform is recorded using a $1 \mathrm{GHz}$ realtime digital oscilloscope (Tektronix, TDS 684B) and averaged over 30 traces. Both lasers have the same circular polarization $\left(\sigma^{+}\right)$. To ensure that linear propagation effect is studied, we keep the intensity of the probe laser, locked to the resonance of the $|1\rangle \rightarrow|3\rangle$ transition, sufficiently low that the atomic population remains primarily in the state $|1\rangle$. The coupling laser is on-resonance with the $|2\rangle \rightarrow|3\rangle$

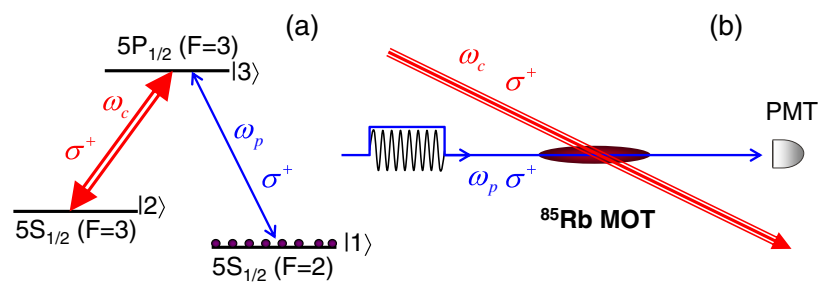

FIG. 1 (color online). (a) ${ }^{85} \mathrm{Rb}$ energy level diagram of a threelevel EIT system. (b) Experimental configuration. 

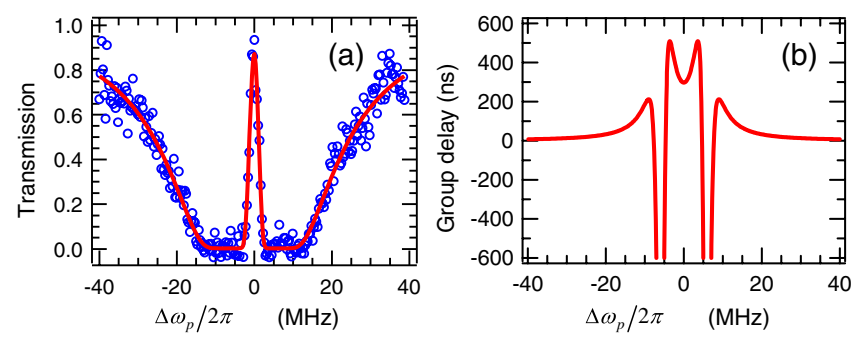

FIG. 2 (color online). (a) Probe laser EIT transmission profile. (b) Group delay as a function of the probe detuning $\Delta \omega_{p} . \alpha_{0} L=$ $45, \Omega_{c}=4 \gamma_{13}$.

transition and renders the medium transparent at the probe resonance frequency. The coupling and probe beams cross each other at the MOT with a $2^{\circ}$ angle. Hence, they are essentially collinear but spatially separated at the PMT.

To understand experimental results, we briefly review the theory of optical pulse propagation. The medium response to the weak probe laser is characterized by its linear susceptibility (complex),

$$
\chi=\frac{\alpha_{0}}{k_{0}} \frac{4\left(\Delta \omega_{p}+i \gamma_{12}\right) \gamma_{13}}{\left|\Omega_{c}\right|^{2}-4\left(\Delta \omega_{p}+i \gamma_{12}\right)\left(\Delta \omega_{p}+i \gamma_{13}\right)},
$$

where $\alpha_{0}$ is the on-resonance absorption coefficient, $k_{0}=$ $\omega_{31} / c, \Delta \omega_{p}=\omega_{p}-\omega_{31}$ is the probe detuning, and $\Omega_{c}$ is the coupling laser Rabi frequency. The atomic lifetime determined dephasing rate between $|1\rangle$ and $|3\rangle$ is $\gamma_{13}=$ $2 \pi \times 3 \mathrm{MHz}$. The measured dephasing rate between the two ground states is $\gamma_{12}=0.01 \gamma_{13}$. When the coupling laser is switched off $\left(\Omega_{c}=0\right)$, Eq. (1) reduces to that of a two-level system. A typical EIT transmission profile is shown in Fig. 2(a). Figure 2(b) shows the calculated group delay as a function of the probe detuning. A large group delay is expected for the frequencies lying in the central narrow EIT window.

Formally, the propagation of a classical pulse through a linear dispersive medium is given by the integral

$$
E(t)=\frac{1}{2 \pi} \int E_{0}\left(\omega_{p}\right) e^{i\left[k\left(\omega_{p}\right) L-\omega_{p} t\right]} d \omega_{p},
$$

where $E_{0}\left(\omega_{p}\right)$ is the spectrum of the initial input pulse, and $k\left(\omega_{p}\right)=k_{0} \sqrt{1+\chi} \simeq k_{0}(1+\chi / 2)$. By using a square pulse with a sufficient length, we can study optical transients at both the rising and falling edges in the same shot. The fast-fourier transform (FFT) is used to numerically evaluate the integral (2). To explain the features of the precursor transients following the edges, it is useful to use the stationary phase method [18]. At high optical depths, the Sommerfeld and Brillouin far-detuned saddle points are in the tails of a Lorentz line, and the medium can be approximated as a two-level system. In this case, the hybrid-asymptotic analysis used in Refs. [6-8,19] gives an analytical expression for the Sommerfeld-Brillouin precursor from a step-modulated pulse $E_{0 \pm}(t)=$ $E_{0} \Theta( \pm t) e^{-i \omega_{0} t}$ as

$$
E_{\mathrm{SB} \pm}(t) \simeq \pm E_{0} J_{0}\left(\sqrt{2 \alpha_{0} L \gamma_{13} \tau}\right) \Theta(\tau) e^{-\gamma_{13} \tau} e^{i\left(k_{0} L-\omega_{0} t\right)}
$$

where $\tau=t-L / c, \omega_{0}=\omega_{31}, J_{0}$ is the 0 -order first kind Bessel function. The \pm signs represent the step-on pulse with a rising edge $(+)$ and the step-off pulse with a falling edge $(-)$, respectively. $\Theta(\tau)$ is the Heaviside function. The main field components within the narrow EIT window travel with a slow light group delay of $\tau_{g} \simeq$ $2 \alpha_{0} L \gamma_{13} /\left|\Omega_{c}\right|^{2}$ and can be approximated as

$$
E_{M \pm}(t) \simeq \frac{E_{0}}{2}\left(1 \pm \operatorname{erf}\left[\frac{\sqrt{\alpha_{0} L}\left(\tau-\tau_{g}\right)}{2 \sqrt{2} \tau_{g}}\right]\right) e^{i\left[k\left(\omega_{0}\right) L-\omega_{0} t\right]} .
$$

The total field is $E_{ \pm}(t)=E_{\mathrm{SB} \pm}(t)+E_{M \pm}(t)$.

There are three characteristic times that determine the observability of optical precursors and its separation from the main field. The first is the oscillation peak duration determined by the Bessel function in Eq. (3) $\tau_{s}=$ $\pi^{2} /\left(2 \alpha_{0} L \gamma_{13}\right)[12,19]$. The second is the precursor (intensity) decay time constant $\tau_{\gamma}=1 /\left(2 \gamma_{13}\right)$ determined by the atomic natural linewidth. The third is the group delay time of the main filed $\tau_{g}$. As discussed in Refs. [12,19], the necessary condition for observing optical precursors is $\tau_{s}<\tau_{g}$. Moreover, to observe precursors with damped

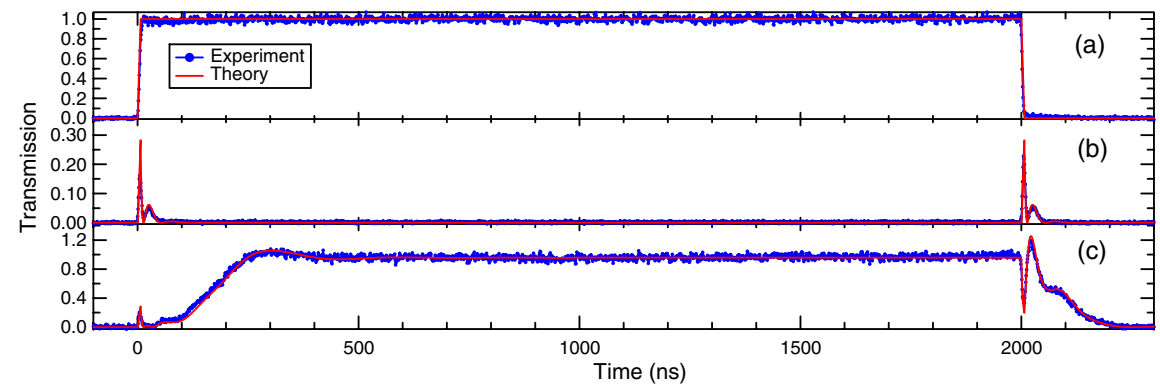

FIG. 3 (color online). Observation of optical precursors from a square-modulated pulse propagating through cold atoms. (a) The reference square pulse with no cold atoms present. (b) $\alpha_{0} L=18$, coupling laser is switched off $\left(\Omega_{c}=0\right)$. (c) $\alpha_{0} L=18$, coupling laser is on $\left(\Omega_{c}=4 \gamma_{13}\right)$. The theoretical curves (red solid line) in (b) and (c) are obtained using FFT by taking into account the finite rise and fall time. 

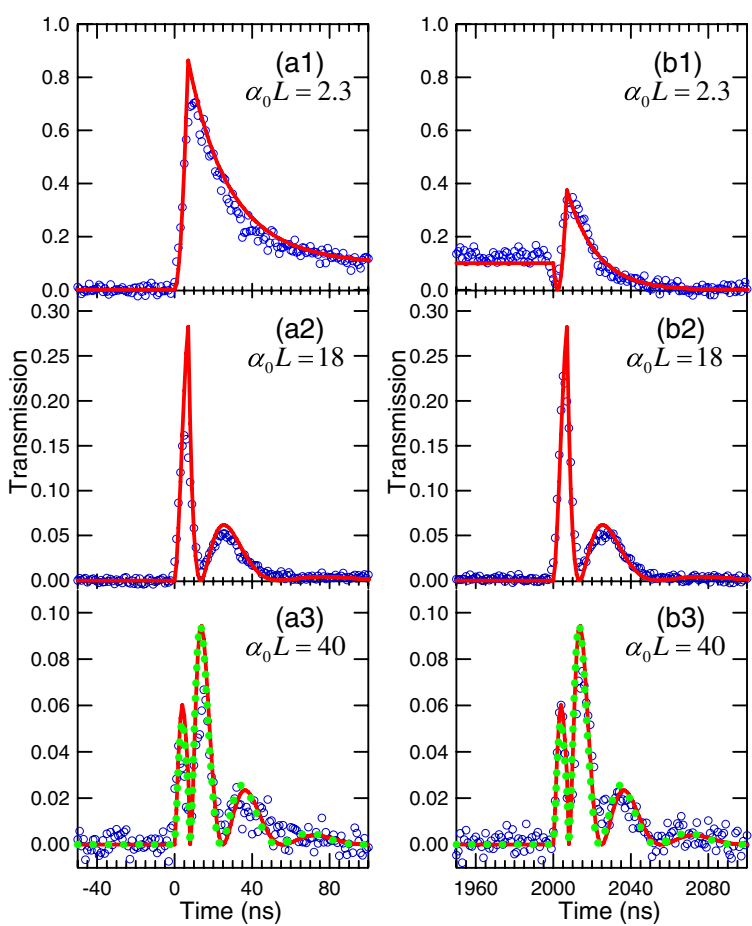

FIG. 4 (color online). Optical precursors at the rising edge [(a1)-(a3)] and falling edge [(b1)-(b3)] from a two-level system at different optical depth. The coupling laser is switched off $\left(\Omega_{c}=0\right)$. The circular (blue) are experimental data. The solid (red) curves are obtained using FFT by taking into account the finite rise and fall time. The dotted (green) points are obtained using hybrid-asymptotic analysis.

oscillatory structure [6-8] well separated from the delayed main field, the characteristic times should satisfy $\tau_{s}<$ $\tau_{\gamma}<\tau_{g}$. With the parameters of the cold atoms, this condition requires optical depth $\alpha_{0} L>\pi^{2} \simeq 10$ and a group delay $\tau_{g}>\tau_{\gamma}=26.5 \mathrm{~ns}$ that are easily met by our experimental system. In this time scale, the finite rise (fall) time of $7 \mathrm{~ns}$ is fast enough to observe the optical precursors.

In our experiment, we first measure precursors in a twolevel system by switching off the coupling laser. As a reference, Fig. 3(a) shows the square pulse measured with no cold atoms present. The experimental result at $\alpha_{0} L=18$ is shown in Fig. 3(b). More details of optical precursors at the rising and falling edges are shown in Fig. 4 for several values of $\alpha_{0} L$ between 0 and 40. As expected according to Sommerfeld and Brillouin, the rising and falling edges travel with speed of light $c$ independent of the atomic density. The transient spikes following the rising and falling edges are optical precursors.

We then repeat the experiments with EIT by turning on the coupling laser. Figure 3(c) shows the output waveform obtained for $\alpha_{0} L=18$ and $\Omega_{c}=4 \gamma_{13}$ for comparison with the results obtained in the two-level system [Fig. 3(b)]. More details of precursors in the EIT system are shown in Fig. 5, for several values of $\alpha_{0} L$ between 0 to 45. As in the two-level case, the rising and falling edges
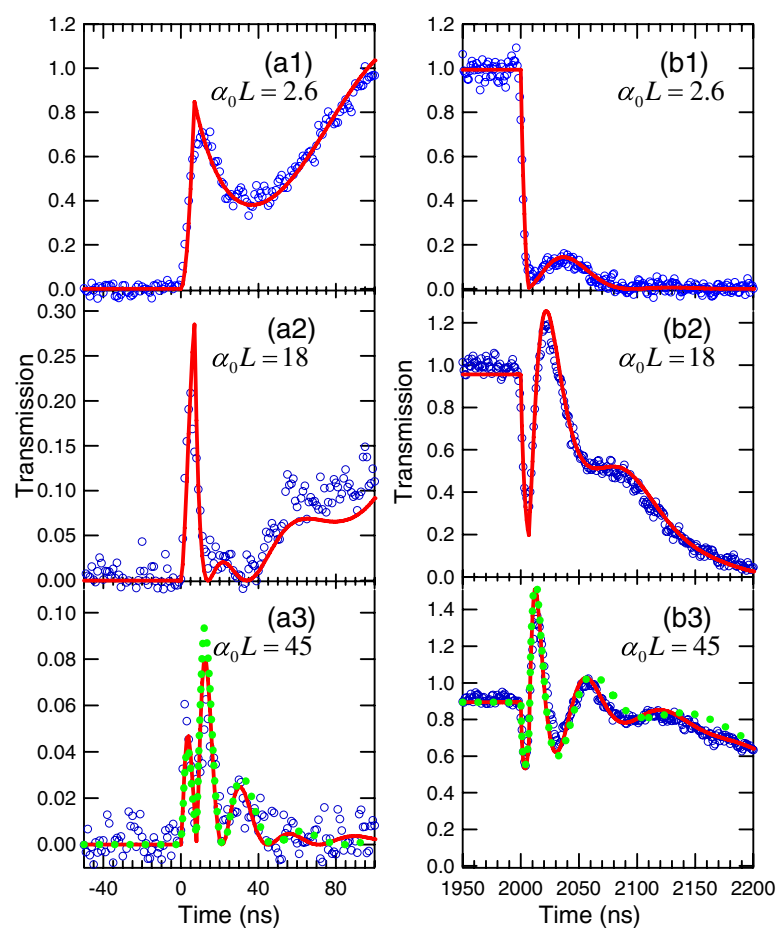

FIG. 5 (color online). Optical precursors at the rising edge [(a1)-(a3)] and falling edge [(b1)-(b3)] from a three-level EIT system at different optical depth and with the same coupling laser power $\left(\Omega_{c}=4 \gamma_{13}\right)$. The circular (blue) are experimental data. The solid (red) curves are obtained using FFT by taking into account the finite rise and fall time. The dotted (green) points are obtained using hybrid-asymptotic analysis.

travel with the same speed of light in vacuum $c$. The main field is delayed with respect to the precursor at the rising edge by a time proportional to $\alpha_{0} L$ due to the EIT slow light effect. At high optical depths $\left(\alpha_{0} L>10\right)$, the precursors are separated from the delayed main pulse. We believe this is the first direct experimental observation showing clearly the separation of optical precursors from the main pulse that is made possible by using an EIT medium. Following the falling edges, damped oscillatory structures are observed.

The data presented in Figs. 4 and 5 are in quantitative agreement with the theoretical results calculated using Eq. (2). The solid (red) curves are calculated using FFT with $1 \mathrm{~ns}$ sampling resolution and for our square input pulse which has finite rise and fall times. One can see that the agreement is very good despite of the fact that there are no adjustable parameters. We now discuss the data by using the hybrid-asymptotic analysis [6,7]. To take into account the finite rise and fall time, we convolve Eqs. (3) and (4) with a square function with a length of 7 ns. For $\alpha_{0} L>10$, the asymptotic analysis gives essentially the same result as that obtained using Eq. (2) and are in good agreement with the data, as shown in the dotted (green) points in (a3) and (b3) of Figs. 4 and 5. However, the 
hybrid-asymptotic analysis allows for a more straightforward interpretation of the various features observed. Figure 4 shows that in the two-level case at high optical depths, the precursors at the rising and falling edges have the same shape and magnitude. This is expected because the main field is absorbed and the precursor, which, according to Eq. (3), should be the same but with a sign difference. Figure 4 also shows that the damped oscillatory precursor pulses has a decay time of about $25 \mathrm{~ns}$, which is consistent with the value of $\tau_{\gamma}$. At low optical depth of $\alpha_{0} L=2.3$, the transient spike at the rising edge has a much larger magnitude than that at the falling edge as a result of the interference between the precursor and the main field. In this case, significant main field is present. Thus, at the rising edge, the main field can interfere with the precursor field constructively. The features in Fig. 5 for the EIT case are qualitatively different. While the precursors at the rising edge have the same shape as those observed in the two-level case, the oscillation at the falling edge appears to decay with a much longer time constant and has a much larger magnitude. Again, all these apparently different features are due to the interference between the main field and the precursor. In the EIT medium, the main field experiences essentially no absorption but is delayed. The group delay times are about 120 and $300 \mathrm{~ns}$ for Fig. 5 (a2) and (a3) and satisfy the condition $\tau_{s}<\tau_{\gamma}<$ $\tau_{g}$. Hence, at the rising edge, there is no interference between the precursor field and the main field, and the precursor waveform should then be essentially the same as those observed in the two-level case. This agrees with the experimental data. At the falling edge, a damped oscillatory structure with $150 \%$ peak transmission caused by the interference between the precursor and delayed main field is observed.

For an ideal step-modulated pulse, the rising (falling) edge is predicted to propagate with zero absorption at all optical depths [6-8]. However, our experimental results show that the precursor signal decreases with increasing optical depth. This is not surprising because all real pulses have finite rise and fall time and they must be properly taken into account in theoretical calculation. When that is done, the agreement between theory and experiment is excellent. From our calculations at $\alpha_{0} L=45$, we note that by reducing the rise time to $1 \mathrm{~ns}$, the transmission of the first peak of the precursor signal increases from 5\% to $65 \%$. This suggests that precursors at still higher optical depths may be observable by using a faster light modulator.

In summary, we have observed optical precursors generated from a long square weak probe pulse propagating through a cold atomic cloud, both in a two-level system, and for the first time, in a three-level EIT system. The optical depth, $\alpha_{0} L$, can be varied from 0 to 50 . In the twolevel case at high optical depth, the main signal is absorbed, and we observe identical precursor transient spikes at the rising and falling edges. In the EIT case, the main signal is not absorbed and travels with a slow group velocity. At the rising edge, the precursor is separated from the main pulse at high $\alpha_{0} L$. At the falling edge, the damped oscillatory structure with $150 \%$ peak transmission is caused by the interference between the precursor and delayed main field. Taking into account the finite rise and fall time of the real square pulse, both FFT and hybridasymptotic theoretical analyses agree well with the experiment. In both the two-level and EIT experiments, the rising and falling edges travel with the speed of light in vacuum. At the optical depth of about 2, there exist negative group delay frequency components with $>10 \%$ transmission. We confirm this by measuring a negative pulse delay of $-50 \mathrm{~ns}$ using a Gaussian pulse with a length of about $350 \mathrm{~ns}$, but we have never measured any observable optical advancement to the rising edge of a square pulse. Therefore, we support that the information velocity does not violate the Einstein causality, and it may be different from the group velocity.

The work was supported by the Hong Kong RGC. Some equipment used in the experiment were provided by the Joyce M. Kuok Lasers and Photonics Laboratory.

*dusw@ust.hk

[1] D. J. Gauthier and R. W. Boyd, Photonics Spectra 82 (2007).

[2] A. Sommerfeld, Ann. Phys. (N.Y.) 44, 199 (1914); L. Brillouin, ibid. 44, 204 (1914).

[3] N. A. Cartwright and K. E. Oughstun, SIAM Rev. 49, 628 (2007).

[4] R. Albanese et al., J. Opt. Soc. Am. A 6, 1441 (1989).

[5] S.-H. Choi and U.L. Österberg, Phys. Rev. Lett. 92, 193903 (2004).

[6] H. Jeong and S. Du, Phys. Rev. A 79, 011802(R) (2009).

[7] B. Macke and B. Segard, Phys. Rev. A 80, 011803(R) (2009).

[8] W. R. LeFew, S. Venakides, and D. J. Gauthier, Phys. Rev. A 79, 063842 (2009).

[9] F. J. Lynch et al., Phys. Rev. 120, 513 (1960).

[10] P. Pleshko and I. Palocz, Phys. Rev. Lett. 22, 1201 (1969).

[11] H. Jeong, A. M. C. Dawes, and D. J. Gauthier, Phys. Rev. Lett. 96, 143901 (2006).

[12] J. Aaviksoo, J. Kuhl, and K. Ploog, Phys. Rev. A 44, R5353 (1991).

[13] E. Varoquaux, G. A. Williams, and O. Avenel, Phys. Rev. B 34, 7617 (1986).

[14] E. Falcon, C. Laroche, and S. Fauve, Phys. Rev. Lett. 91, 064502 (2003).

[15] S. Du, C. Belthangady, P. Kolchin, G. Y. Yin, and S.E. Harris, Opt. Lett. 33, 2149 (2008).

[16] S. E. Harris, Phys. Today 50, No. 7, 36 (1997).

[17] M. Fleischhauer, A. Imamoglu, and J. P. Marangos, Rev. Mod. Phys. 77, 633 (2005).

[18] J.D. Jackson, Classical Electrodynamics (Wiley, New York, 1975), 2nd ed.

[19] J. Aaviksoo, J. Lippmaa, and J. Kuhl, J. Opt. Soc. Am. B 5, 1631 (1988). 